\title{
МЕСТО ТЕХНОЛОГИЙ НЕЙРОМАРКЕТИНГА В ТРАДИЦИОННЫХ МАРКЕТИНГОВЫХ ИССЛЕДОВАНИЯХ
}

\section{THE PLACE OF NEUROMARKETING TECHNOLOGIES IN TRADITIONAL MARKETING RESEARCH}

\section{A. Pismennaya}

Summary: The article deals with traditional research in the field of consumer behavior characteristics. The key features of neuromarketing research and the subject of research are what is important for the customer to know, and what is interesting for the researcher. To accumulate the base and analyze other types of research, the standards and format of the reporting materials are formed taking into account the peculiarities of the research method and the scope. The focus of the study is those factors and parameters that the customer can have a certain impact or change using various marketing techniques and strategies. Specific research objects are studied. The use of systematic and flexible research methods that can be transformed in the course of research, depending on the intermediate results and changing tasks. Each study is always limited by time and budget limits. Comparative analysis of the author showed that the advantage of neuromarketing research is the collection of objective behavioral data using neurophysiological testing and traditional methods of qualitative research. As a result, as an important field of application of neuromarketing, the author identifies a new direction of marketing research.

Keywords: neuroeconomics, neuromarketing, marketing research, methods, market, neuroisearch, method.

\author{
Соискатель, Санкт-Петербургский государственный \\ экономический университет \\ wordsforanna@gmail.com
}

Аннотация: В статье рассматривается традиционные исследования в области особенностей поведения потребителей. Ключевыми особенностям нейромаркетингового исследования и предметом исследования является то, что важно знать заказчику, так и то, что интересно исследователю. Для накопления базы и анализа других видов исследований, стандарты и формат отчетных материалов формируются с учетом особенностей метода исследования и сферы. Фокусом изучения являются те факторы и параметры, на которые заказчик может оказать определенное воздействие или изменить, используя различные техники и стратегии маркетинга. Изучаются конкретные объекты исследования. Применение системных и гибких исследовательских методик, которые можно трансформировать по ходу исследования в зависимости от промежуточных результатов и изменяющихся задач. Каждое исследование всегда ограничено временными и бюджетными рамками. Сравнительный анализ автора показал, что преимуществом нейромаркетингового исследования является сбор объективных поведенческих данных при помощи нейрофизиологического тестирования и традиционных методов качественных исследований. В результате в качестве важной сферы применения нейромаркетинга, автор выделяет новое направление маркетинговых исследований.

Ключевые слова: нейроэкономика, нейромаркетинг, маркетинговые исследования, методы, рынок, нейроисследование, методы.

Особое место в формирующейся нейроэкономике занял нейромаркетинг, изменивший ряд классических представлений о поведении потребителей. Концепция нейромаркетинга была, предположительно, впервые разработана психологами Гарвардского университета (США) в 1990-х годах. Она базируется на утверждении, что за принятие многих потребительских решений в значительной степени отвечает подсознательная, то есть неконтролируемая сознанием область мозга. Это дает возможность изучать данную область в целях «моделирования» поведения потребителей товаров и услуг.

Данная идея предполагает, что "узнавание" возможно в результате объяснения процессов восприятия, памяти и сознания через активность определенных нейронных реакций головного мозга потребителя. При этом конечной задачей нейромаркетинга является более точное понимание поведенческих реакций потребителей, которое позволит эффективнее совершенствовать маркетинговые процессы компании. 
Если взять за основу тезис о том, что человеку как «homo economicus» свойственны полная осведомленность и рациональность, то нейроисследования для рассмотрения вопросов маркетинга и коммуникации являются излишними. Зачем помещать кого-то в МРТ сканер, если можно просто спросить его? Различные эксперименты в области экономической психологии, о которых говорилось выше, показали, что человек не знает всего и не всегда действует рационально, поэтому образ «homo psychologicus» лучше описывает реальность. Решения часто принимаются в бессознательном состоянии и что они частично основаны на эмоциях. Автор придерживается мысли о том, что многие бессознательные ошибки или предубеждения проникли в человеческое мышление и воспоминания, и это означает, что мы делаем меньше рациональных выводов, чем нам кажется. Таким образом, подлинность наших решений может быть поставлена под сомнение, и мы можем даже задаться вопросом, существует ли такая вещь, как свободная воля.

Таким образом, можно сделать вывод, что не на каждый вопрос, который мы устно задаем человеку, может быть дан надежный и достоверный ответ; не из-за злого умысла человека, а потому, что он просто не знает определенных факторов, которые влияют на его ответы. Основываясь на этом аргументе, нейро-исследования заслуживают определенного места в наборе инструментов исследователя рынка.

«Маркетинговые исследования - это вид деятельности, который с помощью информации связывает маркетолога с потребителями, покупателями и общественностью. Полученная информация используется для совершенствования и оценки маркетинговых действий, для отслеживания результатов маркетинговой деятельности, а также для улучшения понимания процесса управления маркетингом» [2. с.313]

В маркетинговых исследованиях используется научный метод познания, предусматривающий сбор и анализ данных для проверки предварительно выдвинутых идей или гипотез. Задача маркетинговых исследований предоставление точной, объективной информации, которая отражает истинное состояние дел. Они должны проводится беспристрастно. И хотя исследование всегда несет отпечаток мировоззрения того, кто его проводит, оно, тем не менее, должно быть свободно от личных или политических пристрастий его самого или руководства.

Исследование, мотивируемое персональной или политической выгодой, нарушает профессиональные нормы. Изначальная предвзятость таких исследований приводит к заранее предопределенным результатам. [3.с.31]

Основной классификацией маркетинговых исследований является деление их на количественные и каче- ственные.

Количественное исследование ставит своей целью получить результаты в количественной форме с использованием методов статистического анализа. Количественное исследование рынка означает изучение видимого поведения. Например: если на товар в течение недели действует скидка, какое количество товара будет продано дополнительно? Этот метод исследования фокусируется на (продажах) цифрах и фактах. Методы поведенческих измерений основаны на «котлеровских моделях» побуждение -реакция. Здесь акцент делается исключительно на том, как наблюдаемое поведение (результат) изменяется в результате определенного стимула. Например: в какой степени изменение контента на сайте привело к увеличению трафика на этом сайте? Многие экспериментальные исследования в области экономической психологии работают следующим образом: распространите запах свежеиспеченного хлеба только в одном из супермаркетов сети и изучите, привело ли это к значительному увеличению продаж хлеба.

Качественное исследование - неструктурированный поисковый метод исследования, основанный на малом объеме выборке, призванный лучше понять обстановку, складывающуюся вокруг проблемы маркетингового исследования. Качественное исследование и направлено на получение данных, не подлежащих статистическому анализу, нацелены на глубокое понимание потребителя, использует для интерпретации полученных результатов не цифры, а слова.

Методы качественного исследования могут быть прямыми или косвенными. Прямые методы, в отличие от косвенных, не скрывают от респондентов истинную цель исследования. Основные прямые методы - это фокус-группы и глубинные интервью. Фокус-группы проводятся в группах, а глубинные интервью с глазу на глаз. Интервью с помощью фокус-группы - это самый распространенный метод качественного исследования. [1.c.209-212]

Качественные методы исследования рынка подходят для поиска объяснений и аргументов. Эти методы имеют кредо: «Мы спрашиваем самих людей». Люди могут рассказать, что им нравится в определенном продукте или услуге и что можно улучшить. Например, жалобы, полученные службой поддержки клиентов, являются хорошим источником информации.

Качественные методы измерения в основном подходят для исследования возможностей изменения ситуации: что идет хорошо и что можно улучшить? Поэтому они позволяют дать рекомендации и оценить результаты. 
Недостатком качественных методов измерения является то, что на результаты исследования влияют всевозможные факторы, которые люди не осознают, как рассматривалось выше. В целом можно сказать, что у качественных методов измерения существует ограничение, заключающееся в том, что они обеспечивают недостаточно эффективное измерение внутренних реакций на внешние раздражители. Эта проблема касается прежде всего изучения будущего поведения потребителей: почти невозможно самому нарисовать объективную картину поведения, которое собираешься продемонстрировать в будущем.

Теперь о нейромаркетинге и его роли в объективности исследований.

Общепризнанным является факт, что один и тот же человек может думать одно, чувствовать другое, говорить третье, а делать совершенно иное. Эта мысль относится к тому факту, что люди допускают определенные мыслительные ошибки при качественных методах измерения и что в своих выражениях они неосознанно руководствуются своим эмоциональным опытом и вещами, которые закреплены в их памяти.

Психофизиологические методы измерения обеспечивают понимание как сознательных, так и бессознательных реакций людей, в результате чего можно обнаружить фактическую (или иррациональную) внутреннюю реакцию на внешние раздражители. Поэтому психофизиологические методы измерения являются подходящим инструментом для отображения эмоционального опыта с брендом или определенным продуктом. Кроме того, эти методы могут быть использованы для решения вопросов, касающихся будущего поведения потребителей. Например, в настоящее время нейромаркетинговые методы измерения используются для проверки потенциальной эффективности рекламы или расширения бренда. Результаты или последствия предложения выбранного маркетингового стимула затем снова измеряют с помощью количественных и качественных методов, и круг, таким образом, завершен. [4.c.304]

На рисунке показано, для какого типа исследовательских вопросов можно использовать тот или иной метод измерения.

Гибридная модель должна рассматриваться как набор инструментов, в котором для разных целей используются разные типы методов. Эти типы методов не могут быть заменены: например, нельзя заменить качественное исследование рынка нейроисследованием. Дело в том, что исследователь имеет четкое представление о конкретном вопросе исследования и выбирает метод измерения, который лучше всего сможет ответить на тот или иной вопрос исследования.

Таблица 1 показывает характер различных исследовательских вопросов в исследовательской матрице в сравнении с различными типами методов. В целом, качественные методы измерения оптимально применять к исследовательским вопросам, касающимся знаний, мнений и пожеланий клиента. Количественные методы измеряют воспринимаемое поведение и могут наилучшим образом отображать предпочтения потребителей. Нейро-исследования в основном измеряют эмоциональный опыт. Поскольку поведение во многом обусловлено этим эмоциональным опытом, нейроисследования мо-

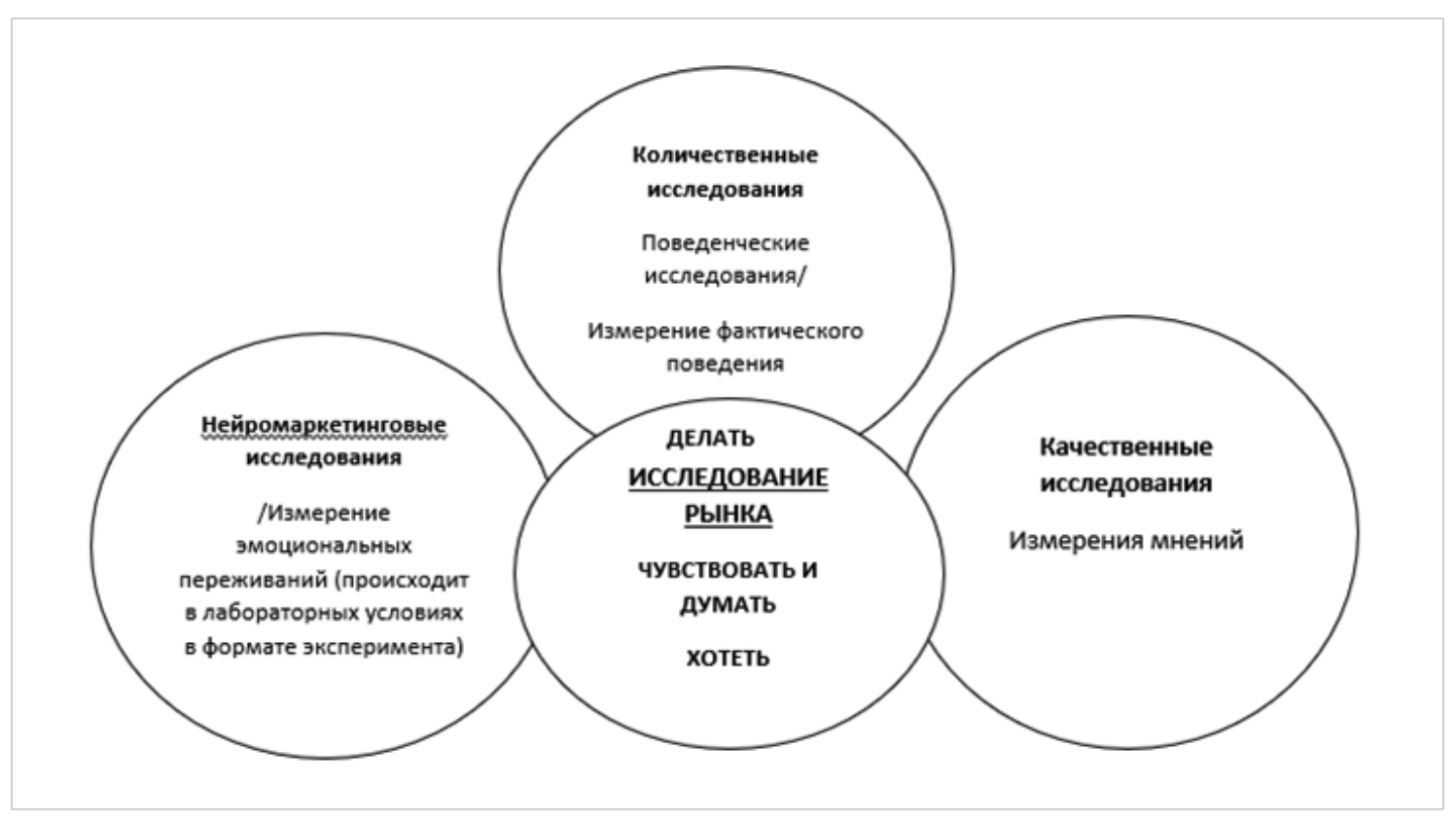

Рис. 1. Виды исследований рынка в зависимости от типа исследовательского вопроса. Источник: Анализ автора. 
гут помочь нарисовать картину будущего поведения потребителя.

Таблица 1.

Матрица исследований: соотношение видов и характера данных

\begin{tabular}{|l|l|l|l|} 
& $\begin{array}{c}\text { Качественные } \\
\text { методы }\end{array}$ & $\begin{array}{c}\text { Количествен- } \\
\text { ные методы }\end{array}$ & $\begin{array}{c}\text { Нейромар- } \\
\text { кетинговые } \\
\text { методы }\end{array}$ \\
\hline Знания & & & \\
\hline Мнения & & & \\
\hline $\begin{array}{l}\text { Желания и потреб- } \\
\text { ности }\end{array}$ & & & \\
\hline Предпочтения & & & \\
\hline Поведение & & & \\
\hline $\begin{array}{l}\text { Эмоциональные } \\
\text { переживания }\end{array}$ & & & \\
\hline Будущее поведение & & & \\
\hline
\end{tabular}

Источник: Анализ автора

Для маркетологов последний пункт в таблице 1 - будущее поведение - имеет решающее значение. В конце концов, люди пытаются направить поведение потребителей в сторону собственного бренда.

Поэтому в контексте маркетинга большое значение имеет прогностическая ценность исследования.

Почему нейромаркетинговые методы измерения в большей степени, чем другие методы, подходят для изучения будущего поведения потребителей? Это связано с ролью отношения человека к объекту, такому как человек, продукт или ситуация. Эти отношения являются результатом мотивации, убеждений и эмоционального опыта человека. Это оценка объекта, и эта оценка может быть положительной или отрицательной. Отношение, как правило, является разумным и хорошим регулятором поведения. Связь между отношением и поведением становится более сильной, когда человек чувствует уверенность, легко может запомнить отношение, основанное на непосредственном опыте. Поэтому нейроисследования являются хорошим инструментом, и могут использоваться для изучения будущего поведения.

Ключевые особенности нейромаркетингового исследования:

- предметом исследования является как то, что важно знать заказчику, так и то, что интересно исследователю для накопления базы и анализа других видов исследований

- стандарты и формат отчетных материалов формируются с учетом особенностей метода исследования и сферы заказчика (который, как правило, не является профессионалом в области нейромаркетинга);

- фокусом изучения являются те факторы и параметры, на которые заказчик может оказать определенное воздействие или изменить, используя различные техники и стратегии маркетинга;

- изучаются конкретные объекты исследования, не усредняя результаты прошлых исследований (за исключением исследований рекламных роликов, и до тех пор, пока не будет накоплена большая база для анализа других видов исследования);

- применение системных и гибких исследовательских методик, которые можно трансформировать по ходу исследования в зависимости от промежуточных результатов и изменяющихся задач;

- исследование всегда ограничено временными и бюджетными рамками.

В настоящее время существуют разные методы проведения качественных маркетинговых исследований потребителей, однако основным критерием является использование социологических методик. В отличие от существующих классических исследований технологии нейромаркетинга позволяют осуществлять сбор и обработку нового вида данных. Специальные приборы дают возможность фиксировать физиологические подсознательные реакции респондентов непосредственно в момент знакомства с упаковкой продукта, ответов на вопросы тестов о продукте или при просмотре визуального материала. Основными параметрами для получения объективных результатов служат такие внутренние факторы потребителей как: внимание, интерес, запоминание, эмоциональная вовлеченность, мотивация к действию. Признаками будут являться учащение пульса и активация реакций определенных участков головного мозга. Данные об этих изменениях можно получить с помощью инструментального и программного обеспечения нейромаркетинга [5 с.114].

По сущностным характеристикам (отвечают на вопрос "Почему?" и "Как?") нейромаркетинговые исследования являются подвидом качественных исследований. Но при накоплении данных можно говорить о том, что нейромаркетинговые исследования также являются подвидом количественных исследований. Для определения преимуществ нейромаркетинговых исследований автором был проведен сравнительный анализ качественных и нейромаркетинговых исследований. (Таблица 2)

Сравнительный анализ показал, что преимуществом нейромаркетингового исследования является сбор объективных поведенческих данных при помощи нейрофизиологического тестирования и традиционных методов качественных исследований. Нейромаркетинговые исследования могут являться самостоятельным видом исследования, но их эффективность существенно выше, 
Сравнительный анализ качественных и нейромаркетинговых исследований

\begin{tabular}{|c|c|c|}
\hline Критерии & $\begin{array}{c}\text { Традиционные качественные исследования (фокус- } \\
\text { групповые дискуссии, интервью) }\end{array}$ & Нейромаркетинговые исследования \\
\hline Специфика исследования & $\begin{array}{l}\text { Описать поведение, которое исследуемые осознают на } \\
\text { вербальном уровне } \\
\text { Выявить реакции на те или иные инструменты }\end{array}$ & $\begin{array}{l}\text { Описать реакцию и поведение, которое исследуемые } \\
\text { могут не осознавать во время теста разных маркетин- } \\
\text { говых стимулов }\end{array}$ \\
\hline Базовые техники и технологии & $\begin{array}{l}\text { В основе исследования - интервью потребителя, от- } \\
\text { крытые вопросы, субъект-субъектное взаимодействие }\end{array}$ & $\begin{array}{l}\text { Техника применения айтрекера, полиграфа, камера } \\
\text { для фиксации мимики лица и электроэнцефалограф } \\
\text { энцефалографа с дальнейшим анализом данных. } \\
\text { Взаимодействие «человек - машина» }\end{array}$ \\
\hline Степень субъективности результатов & $\begin{array}{l}\text { Позволяют выявить субъективные предпочтения } \\
\text { определенной категории людей, что лишает возмож- } \\
\text { ности обобщения }\end{array}$ & $\begin{array}{l}\text { Анализ результатов происходит на основе нейрофизи- } \\
\text { ологических данных, что позволяет заявлять } 06 \text { адап- } \\
\text { тивности результатов к разным категориям людей }\end{array}$ \\
\hline $\begin{array}{l}\text { Влияние лидеров мнений в изучаемых } \\
\text { группах }\end{array}$ & $\begin{array}{l}\text { Сильное влияние может оказать лидер мнения при } \\
\text { проведении групповых дискуссий }\end{array}$ & $\begin{array}{l}\text { Анализ результатов происходит на основе нейрофизио- } \\
\text { логических данных каждого респондента, что снижает } \\
\text { вероятность «навязанного ответа» }\end{array}$ \\
\hline Затраты на исследования & Высокая стоимость исследования & $\begin{array}{l}\text { Стоимость исследования не выше, чем традиционные } \\
\text { качественные исследования }\end{array}$ \\
\hline Типы собираемых данных & $\begin{array}{l}\text { Позволяют получить данные только вербальных осоз- } \\
\text { нанных оценок респондентов. } \\
\text { Субъективные данные }\end{array}$ & $\begin{array}{l}\text { Используют комплексный подход сбора поведенческих } \\
\text { данных, который включает виды традиционных каче- } \\
\text { ственных исследований (фокус-групповая дискуссия, } \\
\text { анкетирование или интервью) после проведения ней- } \\
\text { рофизиологических замеров. } \\
\text { Субъективные и объективные данные }\end{array}$ \\
\hline Методы сбора данных & $\begin{array}{l}\text { Фокус-группы, интервью, тестирования, наблюдения и } \\
\text { т.д. }\end{array}$ & Замеры нейрофизиологических реакций \\
\hline Методы обработки данных & $\begin{array}{l}\text { Описание связей между изучаемыми переменными, } \\
\text { проверка гипотез и т.д. }\end{array}$ & $\begin{array}{l}\text { Интерпретация динамических и статических данных. } \\
\text { Кодирование лицевых значений, обработка данных } \\
\text { динамики реакций головного мозга, метод синхронной } \\
\text { регистрации параметров дыхания, сердечно-сосуди- } \\
\text { стой активности и электрического сопротивления кожи }\end{array}$ \\
\hline
\end{tabular}

Источник: Анализ автора

когда они используются в дополнение к традиционным методам.

Именно комплексный подход позволяет получить максимально допустимый массив данных о потребительской реакции на тот или иной маркетинговый материал. Тем самым маркетолог получает возможность не только использовать результат, но и определить причины данного результата.

В условиях массовой коммуникации для эффективного донесения маркетинговой информации, выраженной рекламным сообщением или описанием продукта на упаковке, помимо прочих условий, необходимо одинаковое понимание символов теми, кто их воспринимает. Информация должна быть понятна и в содержательном, и в языковом аспекте.

Оригинальность данного подхода состоит в том, что при помощи нейромаркетинговых исследований в отличие от существующих методик можно получить более точные ответы о том, какие эмоции испытывают потребители при контакте с исследуемым продуктом, а также однозначно определить их предпочтения среди представленных для выбора вариантов. Для того, чтобы определить причины той или иной оценки респондентов, необходимо дополнить их опросом или интервьюированием. Сравнивая ответы «до и во-время» использования специальных приборов, становится доступным составление целостной картины оценки потребителем исследуемого материала.

Преимуществом нейромаркетингового исследования является сбор объективных поведенческих данных, при помощи нейрофизиологического тестирования и традиционных методов качественных исследований. Именно комплексный подход позволяет получить максимально допустимый массив данных о потребитель- 
ской реакции на тот или иной маркетинговый материал. Тем самым, маркетолог получает возможность не только получить результат, но и определить причины данных результатов.

В результате в качестве важной сферы применения нейромаркетинга автор выделяет маркетинговую аналитику, учитывающую внутренние факторы поведения потребителей и позволяющую строить прогноз потребительского решения. Возможность регистрации нейробиологических процессов принятия решений позволяет улучшить параметры исследуемого продукта и определить тренд потребительских решений на всех этапах их формирования. Выделяя внутренние факторы потребителей как ключевые объекты исследования, нейромаркетинг формирует новый вид исследований - нейромаркетинговое тестирование. Преимуществом данного типа тестирования является совмещение методов нейромаркетинга и классических методов качественного исследования, что позволяет определить комплексную оценку респондентами стимульного материала. В классическом исследовании респондент сообщает сформулированный ответ, где первичные эмоции и неосознанные мотивы проходят через «фильтр формулирования». То есть респондент может за формулировкой ответа скрыть свою подлинную оценку, а также изменить свое мнение под воздействием других участников исследования или интервьюера. При помощи данных, полученных от приборов тестирования, не всегда можно определить причину того или иного решения об исследуемом материале. В таком случае следует проводить фокус-групповую дискуссию после фиксации эмоциональных реакций респондента для объяснения причин того или иного решения. Для определения преимуществ нового вида исследований автором предлагается уточнить понятие «нейромаркетинговые исследования».

Оценить силу воздействия, понять, о чем думает человек в момент контакта с маркетинговыми стимулами - задача инструментального нейромаркетинга. Его возможности позволяют проводить исследования в области ценообразования, поведения потребителя у полки в магазине с имитацией реального процесса покупки, оценивать любые рекламные носители: сайт, упаковку, билборд, видео, фильм, компьютерные игры, слоганы, логотипы и многое другое.

После оценки объема данных, полученных с помощью специального оборудования, разрабатываются технические руководства для дизайна, программирования и стратегического развития маркетинга. Они в свою очередь создают рекламные материалы, которые бы пробуждали необходимые эмоции, воздействуя через обоняние, зрение, вкус, осязание, и вели потребителя к покупке.

Нейромаркетинговые исследования - это новое направление маркетинговых исследований, предметом которого является сбор, анализ и интерпретация данных о физиологических подсознательных реакциях человека на определенные стимулы с целью совершенствования маркетинговых технологий, программ, стратегий, а также построения тренда покупательского поведения. В отличие от традиционных методов исследования нейромаркетинговые позволяют осуществлять сбор нового вида данных как: внимание, интерес, запоминание, эмоциональная вовлеченность, мотивация к действию.

\section{ЛИТЕРАТУРА}

1. Ассэль, Г. Маркетинг: принципы и стратегия, М.: Инфра-М, 2002.

2. Ф. Котлер Основы маркетинга. Изд. Вильямс 2002

3. Малхотра, Нэреш К. Маркетинговые исследования. Практическое руководство, 3-е издание.: Пер. с англ. - М.: Издательский дом "Вильяме", 2002.

4. Льюис Д. Нейромаркетинг в действии. Как проникнуть в мозг покупателя. - М.: Манн, Иванов и Фербер, 2015 г.

5. Трайндл А. Нейромаркетинг: Визуализация эмоций / пер. с нем. А. Гордеева - М.: Альпина Бизнес Букс, 2007.

6. Glimcher Paul W. EN. Foundations of Neuroeconomic Analysis. - Gardners Books, 2011.

(c) Письменная Анна Сергеевна (wordsforanna@gmail.com). 